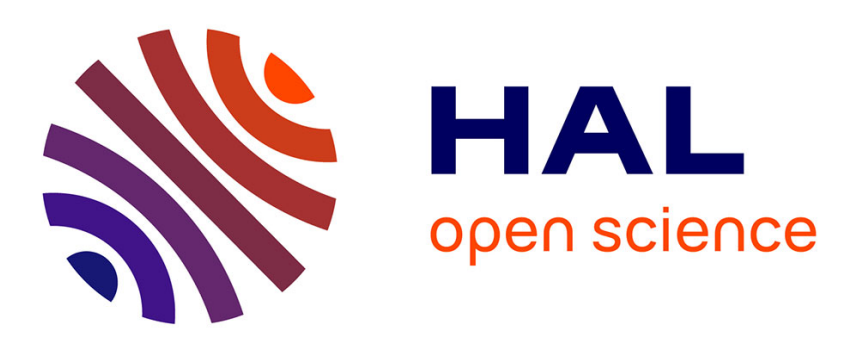

\title{
Thresholded Multiple Coherence as a tool for source separation and denoising: Theory and aeroacoustic applications
}

Quentin Leclere, Alice Dinsenmeyer, Jérôme Antoni, Emmanuel Julliard, Azucena Pintado-Peño

\section{To cite this version:}

Quentin Leclere, Alice Dinsenmeyer, Jérôme Antoni, Emmanuel Julliard, Azucena Pintado-Peño. Thresholded Multiple Coherence as a tool for source separation and denoising: Theory and aeroacoustic applications. Applied Acoustics, 2021, 178, pp.108021. 10.1016/j.apacoust.2021.108021 . hal03169204

\section{HAL Id: hal-03169204 \\ https://hal.science/hal-03169204}

Submitted on 15 Mar 2021

HAL is a multi-disciplinary open access archive for the deposit and dissemination of scientific research documents, whether they are published or not. The documents may come from teaching and research institutions in France or abroad, or from public or private research centers.
L'archive ouverte pluridisciplinaire HAL, est destinée au dépôt et à la diffusion de documents scientifiques de niveau recherche, publiés ou non, émanant des établissements d'enseignement et de recherche français ou étrangers, des laboratoires publics ou privés. 


\title{
Thresholded Multiple Coherence as a tool for source separation and denoising: theory and aeroacoustic applications
}

\author{
Quentin Leclère $^{\mathrm{a}, *}$ Alice Dinsenmeyer ${ }^{\text {a }}$ Jérôme Antoni ${ }^{\mathrm{a}}$ \\ Emmanuel Julliard ${ }^{b}$ Azucena Pintado-Peño ${ }^{b}$

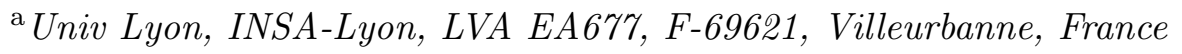 \\ ${ }^{\mathrm{b}}$ Airbus Operations S.A.S, Acoustics Department, Toulouse, France
}

\begin{abstract}
The multiple coherence is a spectral analysis tool allowing the estimation of the contribution of several, possibly partially, coherent inputs to one or several outputs. This type of analysis can be conducted using a waterfall substraction approach (Conditioned Spectral Analysis framework) or using an eigenvalue analysis of the input correlation matrix (Virtual Source Analysis approaches). Those techniques are well established when dealing with converged cross-spectral estimates. In practice, this is never the case because of the finite nature of time records, and it can bring interpretation issues, particularly when increasing the number of references. The significance of the estimated coherence plays a central role in the present work. It involves the implementation of an hypothesis test based upon the statistical behavior of the estimated coherence between incoherent signals. This test, whose principle is to put to zero an estimated coherence that is below a significance threshold, is extended in this work to the multiple coherence case. The TMC (Thresholded Multiple Coherence) is first illustrated in the frame of a numerical benchmark, and then validated in a laboratory wind tunnel test where the interest for denoising purpose is demonstrated. The approach is finally applied to signals recorded inside and outside the cabin of an aircraft during a flight test. The TMC is used either from outside to inside microphones, to analyse the contribution of outside noise sources to the interior noise, or alternatively from inside to outside sensors, for flow noise rejection purpose.
\end{abstract}

Key words: thresholded coherence, multiple coherence, denoising, source separation, flight tests

\footnotetext{
* Corresponding author. Fax: +33.4.72.43.87.12. E-mail address: quentin.leclere@insa-lyon.fr.
} 


\section{Introduction}

The coherence is a frequency-dependent function estimating the level of linear dependency between two signals. It is directly related to the well known Cauchy-Schwarz inequality

$$
|\langle\mathbf{X}, \mathbf{Y}\rangle|^{2} \leq\langle\mathbf{X}, \mathbf{X}\rangle\langle\mathbf{Y}, \mathbf{Y}\rangle
$$

where $\mathbf{X}, \mathbf{Y}$ are two vectors $\in \mathbb{C}^{N}$ and $\langle\mathbf{X}, \mathbf{Y}\rangle=\sum_{n=1}^{N} X_{n} \overline{Y_{n}}$ the standard hermitian scalar product. In spectral analysis, $\mathbf{X}$ and $\mathbf{Y}$ contain the Fourier coefficients of the two signals of interest $x(t)$ and $y(t)$ at a given frequency, obtained for $N$ finite realizations (called snapshots). The estimated coherence is simply defined as the ratio between the left and right hand side of Eq. (1), thus lying between 0 (orthogonality) and 1 (colinearity). An estimated coherence value that is significantly greater than zero at a given frequency indicates the existence of a linear relationship between the two signals, that can be interpreted in vibration and acoustics as a filter or a transfer path. The question of the significance is of prime interest : it is clear that if signals are fully dependent, the estimated coherence will be equal to one even for low values of $N$ (because of the colinearity of $\mathbf{X}$ and $\mathbf{Y}$ ). However, if the actual coherence value is low, the estimation error has to be considered [1]. In the particular case of independent signals, the coherence will be systematically overestimated since $\mathbf{X}$ and $\mathbf{Y}$ will never be fully orthogonal. As an illustration, Fig. 1 gives some coherence functions estimated by the Welch's periodogram $[2]$ between two independent gaussian time series of different durations. The theoretical coherence should be 0 on the whole frequency range, but due to the finite length of signals it is not the case.

It is possible to define statistically a significance threshold, in order to au-
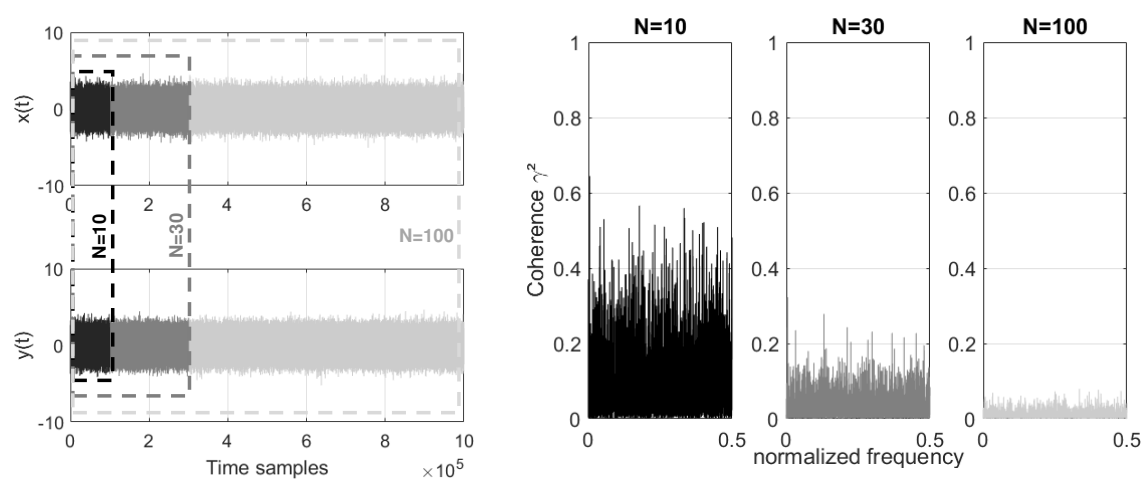

Fig. 1. Time data (left) and coherence estimate (right) for two independent signals $x(t)$ and $y(t)$ of different finite lengths ( $\mathrm{N}$ stands for the number of snapshots).

tomatically determine if the coherence is significantly non zero [3]. However, this possibility has received only few attention in the literature; one can cite 
at least the work of Miles on turbofan engine noise [4], and the contribution of Gallet and Julien [5] in the biomedical domain.

Once a coherence is established between $x(t)$ and $y(t)$, and considering that $x(t)$ is an input and $y(t)$ an output, it is possible to determine the contribution of $x(t)$ to $y(t)$. In the Fourier domain, this contribution is defined as the Coherent Output Power (COP), introduced in the 70's [6], it results directly from the product between the coherence and the power spectrum of the output. In multiple input situations, this approach is limited by the potential correlation between inputs, making it difficult to separate contributions of inputs to output(s). This issue led to the development of more advanced analysis techniques, for instance partial and multiple coherence approaches $[7,8,9]$, or virtual source analysis $\mid 10,11,12\rfloor$, that have proven some efficiency in several industrial applications $[13,14,15,16]$.

Coherence-based methods have been used especially in the field of aeroacoustics $[17,18,19,20]$, which can be explained by the fact that aeroacoustic sources are often physically distributed with relatively short coherence lengths, facilitating their separation using statistical approaches. The difficulty is then to be able to represent the source of interest with a sufficient number of references, in a context where the SNR can be strongly affected by flow noise.

The originality of the present work is to propose the implementation of a coherence thresholding step in a multiple input context. The thresholding of the coherence is not a new concept $[3,4,5]$, but it has never been addressed, to our knowledge, to the case of the multiple coherence. Another contribution of the paper is to demonstrate the potential use and efficiency of the so-called TMC (Thresholded Multiple Coherence) within two experiments in the field of aeronautics, a first one in laboratory conditions (wind tunnel) and a second one in the frame of an industrial application (flight tests).

The first section is dedicated to theoretical aspects of the TMC, with the description of the hypothesis test so as to determine if a coherence is (or is not) significantly greater than 0 . An approach relying on the eigenvalue decomposition of the reference cross spectral matrix is then proposed to adapt the test to the multiple coherence. A numerical experiment is proposed in a second section, so as to illustrate the efficiency of the TMC for several varying parameters: SNR of references, number of references, and risk value associated to the hypothesis test. A laboratory experiment is reported in the third section of the paper, demonstrating the pertinence of the approach for denoising applications in a wind tunnel. Finally, the TMC is implemented to analyse microphone signals recorded during aircraft flight tests. Several microphones are spatially distributed inside the cabin and outside on the fuselage of the aircraft. The TMC can be used to either estimate the contribution of external noise sources to the interior noise (in this case the references are microphones 
distributed on the fuselage, with a poor SNR due to the turbulent boundary layer that is developed on the fuselage), or to remove from fuselage microphones the contribution of the turbulent boundary layer (in this case the references are microphones placed inside the cabin).

The basic idea of the thresholded multiple coherence has been initially proposed in a former conference paper $\lfloor 21\rfloor$ by the authors of the present work. The present paper develops the full theory behind the TMC, and illustrates the concept with numerical and experimental application cases.

\section{Theoretical aspects}

\subsection{Probability laws of the estimated coherence between uncorrelated signals}

Let $x(t)$ and $y(t)$ be two uncorrelated signals. Let $\mathrm{X}$ and $\mathrm{Y}$ be the Fourier coefficients of one finite length realization of these two signals at a given frequency $f$ (excluding 0 and Nyquist frequency bins), $\mathrm{X}$ and $\mathrm{Y}$ are random variables following centered complex Gaussian laws. The estimated coherence is defined as the following ratio (variable $f$ is dropped for the sake of notation simplicity)

$$
\gamma_{x y}^{2}=\frac{\left|S_{x y}\right|^{2}}{S_{x x} S_{y y}}
$$

where $S_{x x}, S_{y y}$ and $S_{x y}$ are auto and cross-spectra averaged over $N$ finite realizations of the signals:

$$
S_{x x}=\frac{1}{N} \sum_{i=1}^{N}\left|X_{i}\right|^{2} ; S_{y y}=\frac{1}{N} \sum_{i=1}^{N}\left|Y_{i}\right|^{2} ; S_{x y}=\frac{1}{N} \sum_{i=1}^{N} X_{i} \overline{Y_{i}} .
$$

The probability law of the estimated coherence of uncorrelated Gaussian signals is given by Carter [22]. The associated PDF (Probability Density Function) is:

$$
\begin{aligned}
f_{\gamma_{x y}^{2}}(z)=(N-1)(1-z)^{N-2} & \text { for } 0<z<1, \\
f_{\gamma_{x y}^{2}}(z)=0 & \text { for } z<0 \text { or } z>1,
\end{aligned}
$$

with the following mean value and variance

$$
E\left[\gamma_{x y}^{2}\right]=\frac{1}{N} \quad ; \quad V\left[\gamma_{x y}^{2}\right]=\frac{1}{N^{2}} \frac{N-1}{N+1} .
$$

This probability law is valid for non overlapping time segments of signals $x(t)$ and $y(t)$. In practice, using some overlap between consecutive time segments 
is advised, associated to an apodization window, so as to optimize the convergence of estimators for a given total record length. By doing so, the number of averages (noted $N$ ) can artificially increase (if a strong overlap is used), but some redundancy is created between time snapshots. The standard $1 / N$ rule for the variance of quadratic spectral estimates does not stand anymore. However, this can be corrected by replacing the real number of snapshots $N$ by an apparent number of snapshots :

$$
N_{a p}=\frac{N}{c_{W}},
$$

where $c_{W}$ is a correction depending on the overlap value and the window type $[2,5]$. If the overlap value is such that $1 /(1-$ overlap $)$ is an integer, which is the case for classical overlap values $(50,67,75 \%)$, so as to use the same total record length, the apparent value of the number of snapshots can be expressed as a function of the number of averages without overlap, noted $N_{0}$ :

$$
N_{a p}=\frac{1}{c_{W}}\left(\frac{N_{0}-1}{1-\text { overlap }}+1\right) .
$$

The resulting ration between $N_{a p}$ and $N_{0}$ is drawn in Fig. 2 for different window types, $N_{0}$ and overlap values. It can be seen in particular that using a
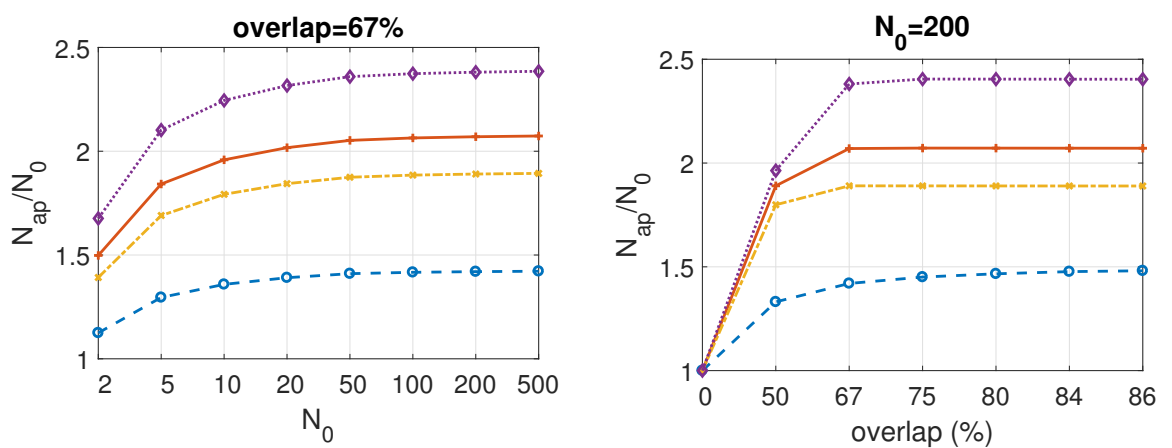

Fig. 2. Left: ratio $N_{a p} / N_{0}$ as a function of $N_{0}$, overlap fixed at $67 \%$. Right: ratio $N_{a p} / N_{0}$ as a function of the overlap, $N_{0}$ fixed at $100 \%$. Window types : Uniform (dashed blue o), Hanning (solid red + ), Hamming (dash dot yellow $\times$ ), Blackmann $($ dotted purple $\diamond)$

Hanning window, an overlap of $67 \%$ and a number of snapshot without average larger than 100 then an asymptotic value of about 2.07 is reached.

Gallet [5] proposes to replace the real number of snapshots by the apparent value so as to represent correctly the statistical behavior of the estimated coherence of independent signals. Some numerical simulation have been conducted to illustrate the validity of this approximation. The coherence between two independent signals is calculated with an overlap value of $67 \%$, hanning window, and different values of $N_{0}$. Results are drawn as histograms in Fig. 3 , together with the theoretical PDF (Eq. (4)) replacing $N$ by $N_{a p}$ (Eq. (6)). This approximation is known to slightly differ from the real distribution only 

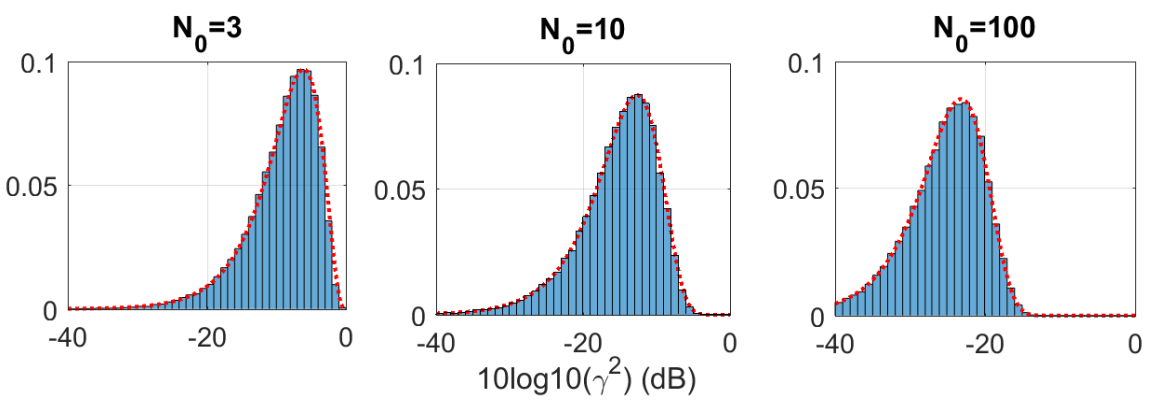

Fig. 3. Distributions resulting from numerical draws (histograms) and theoretical PDF (with the approximation $N=N_{a p}$ ) of the estimated coherence (in dB) of independent signals, Hanning window, $67 \%$ overlap, for different values of $N_{0}$.

for very low values of $N_{0}[5]$. For $N_{0}=3$, some differences can indeed be seen between the histogram and the theoretical distribution. However, for higher values of $N_{0}=10$ or 100, no noticeable differences are observed. Consequently, the apparent value of the number of snapshots $\left(N_{a p}\right)$ is used in the following in place of the true value of $N$.

\subsection{A hypothesis test for the significance of the coherence}

The probability law of the coherence (Eq. (4)), together with the correction of $N$ due to windowing and overlap (Eq. (7)), can be used to implement a statistical test in order to decide if the two signals can be assumed as fully uncorrelated or not. Let's assume that the two signals are fully uncorrelated $\left(\mathrm{H}_{0}\right.$ hypothesis), there is an user-defined small probability $\alpha$ (risk of the test) that the coherence is greater than a given threshold depending on $\alpha$ and $N_{0}$. If the observed value is smaller than the threshold, the coherence is considered as not significantly different from 0 (signals are uncorrelated). If the observed value is greater than the threshold, the $H_{0}$ hypothesis is rejected, the value of the estimated coherence is kept as it is. From Eq. (4), the threshold $T$ is defined by $[4]$

$$
p\left(\gamma_{x y}^{2}<T\right)=\int_{0}^{T}\left(N_{a p}-1\right)(1-z)^{N_{a p}-2} d z=1-\alpha,
$$

which gives

$$
T\left(\alpha, N_{a p}\right)=1-\alpha^{1 /\left(N_{a p}-1\right)} .
$$

The threshold is given in table 1 , as an illustration, for different values of $\alpha$ and $N_{0}$, with a Hanning window and an overlap equal to $67 \%$.

Values in Table 1 correspond to the minimal values leading to a decision that the coherence is not zero. For instance, with a risk value of $1 \%$, the approach will consider that a coherence of $10 \%$ is not significantly greater than 0 for $N_{0}$ equal to 20 . If the same coherence of $10 \%$ is observed with $N_{0}$ equal to 50 , then the coherence is considered as significant. 
Table 1

\begin{tabular}{|c|c|c|c|c|}
\hline$N_{0}$ & $N_{a p}$ & $\alpha=5 \%$ & $\alpha=1 \%$ & $\alpha=0.1 \%$ \\
\hline 20 & 40.3 & 0.073 & 0.11 & 0.16 \\
\hline 50 & 102.6 & 0.029 & 0.044 & 0.066 \\
\hline 200 & 413.7 & 0.0072 & 0.011 & 0.017 \\
\hline 500 & 1036.4 & 0.0029 & 0.0044 & 0.0066 \\
\hline
\end{tabular}

Threshold T such that $p\left(\gamma_{x y}^{2}>T \mid H_{0}\right)=\alpha$, Hanning window, overlap $67 \%$.

Formally, the thresholded coherence function can be expressed as follows :

$$
\gamma_{x y, \alpha}^{2}=\mathbf{1}_{\left[\gamma_{x y}^{2}>T\left(\alpha, N_{a p}\right)\right]} \cdot \gamma_{x y}^{2}
$$

where $\mathbf{1}_{\text {[statement] }}$ is equal to 1 or 0 if statement is true or false, respectively.

\subsection{The coherent output power for SISO and MISO cases}

Let $x(t)$ and $y(t)$ be two signals recorded simultaneously from a given experimental case. Assuming that $x$ is an input, the Coherent Output Power (COP) of $y$ (with respect to $x$ ) is defined by:

$$
S_{y y}^{(x)}=\gamma_{y x}^{2} S_{y y}
$$

and can be interpreted as the energy of $y$ that is linearly explained by $x$ at the frequency of interest. If $x$ represents a source signal, $S_{y y}^{(x)}$ is interpreted as the contribution of $x$ to $y$. The COP is in fact related to the $\mathrm{H} 1$ estimator of the Frequency Response Function $Y / X$ that assumes that the noise contaminates the output signal only:

$$
S_{y y}^{(x)}=\gamma_{y x}^{2} S_{y y}=\frac{\left|S_{y x}\right|^{2}}{S_{x x} S_{y y}} S_{y y}=\frac{\left|S_{y x}\right|^{2}}{S_{x x}}=\left|\mathrm{H} 1_{y x}\right|^{2} S_{x x}
$$

where $\mathrm{H} 1_{y x}=S_{y x} / S_{x x}$. It is clear using this formulation that the COP represents the contribution of $x$ to $y$, the remaining part of $S_{y y}$ (equal to $\left.\left(1-\gamma_{y x}^{2}\right) S_{y y}\right)$ representing the contribution of the noise. In this context, the interest of thresholding the coherence is straightforward : if the estimated coherence is not significantly greater than 0 , then the contribution of $x$ to $y$ is considered as null.

In some situation it can be interesting to estimate the contribution of $n$ reference signals, $a(t), b(t), c(t), \ldots \mu(t)$ to the output $y(t)$ (MISO case for Multiple Input Single Output). However, reference signals are rarely uncorrelated, such that the energy of the output cannot be simply defined as the sum of the COPs with respect to each reference. Bendat and Piersol proposed 40 years ago $[9]$ to proceed iteratively, reference by reference (Conditioned Spectral 
Analysis - CSA framework, that can also be interpreted as a Gram-Schmidt orthogonalization process). The COP is firstly calculated with respect to $a$ :

$$
S_{y y}^{(a)}=\gamma_{y a}^{2} S_{y y}=\frac{\left|S_{y a}\right|^{2}}{S_{a a}}
$$

Then, the contribution of the first reference is removed from auto and cross spectra of other channels :

$$
S_{i j . a}=S_{i j}-\frac{S_{i a} S_{a j}}{S_{a a}}, \forall i, j \in\{y, b, c \ldots \mu\}
$$

$S_{i j . a}$ are called auto and cross spectra conditioned by $a$. The second step is to calculate a COP with respect to a second reference $b$ using quantities conditioned by $a$ :

$$
S_{y y . a}^{(b)}=\frac{\left|S_{y b . a}\right|^{2}}{S_{b b . a}}
$$

The contribution of the second reference is removed to obtain cross spectral quantities conditioned by $a$ and $b$ :

$$
S_{i j . a, b}=S_{i j . a}-\frac{S_{i b . a} S_{b j . a}}{S_{b b . a}}, \forall i, j \in\{y, c, \ldots \mu\}
$$

and so on up to the last reference. At the end, the multiple coherent output power (MCOP) can be obtain as follows :

$$
S_{y y}^{(a, b, \ldots \mu)}=S_{y y}^{(a)}+S_{y y \cdot a}^{(b)}+S_{y y \cdot a, b}^{(c)}+\ldots . S_{y y \cdot a, b, \ldots}^{(\mu)},
$$

the multiple coherence corresponding to the ratio between this result and the total output power :

$$
\Gamma_{y}^{2}=\frac{S_{y y}^{(a, b, \ldots \mu)}}{S_{y y}} .
$$

This multiple coherence can be interpreted as the part of the output signal that can be linearly explained by the set of references. If the references are representing sources, the multiple coherent output power can be interpreted as the contribution of the referenced sources to the output signal.

An alternative to Conditioned Spectral Analysis for the estimation of the multiple coherence is obtained through the Virtual Source Analysis (VSA), proposed by Price and Bernhard [10] in the 80's. This approach leads theoretically to the same result for the multiple coherence than CSA, yet it is more suited for the further developments proposed in present work. VSA is based on an eigenvalue decomposition of the cross spectral matrix of the reference signals. Let $\lambda_{v}^{2}$ be the $v^{\text {th }}$ (real positive) eigenvalue and $\Phi_{j v}$ the $j^{\text {th }}$ element of the $v^{t h}$ eigenvector $(v, j \in[1 \ldots n])$. Eigenvalues can be interpreted as a set of $n$ uncorrelated virtual source powers contributing to reference channels. The 
cross spectrum between the output and the $v^{t h}$ virtual source is:

$$
S_{v y}=\sum_{j=1}^{n} \overline{\Phi_{j v}} S_{j y}
$$

The virtual coherence represents the coherence between any virtual source and the output:

$$
\gamma_{v y}^{2}=\frac{\left|S_{v y}\right|^{2}}{S_{v v} S_{y y}}=\frac{1}{\lambda_{v}^{2} S_{y y}}\left|\sum_{j=1}^{n} \overline{\Phi_{j v}} S_{j y}\right|^{2},
$$

with a corresponding virtual COP given by

$$
S_{y y}^{(v)}=\gamma_{v y}^{2} S_{y y}
$$

The estimated coherence between virtual sources have the property to be zero, such that their contributions to $y$ can simply be added energetically :

$$
S_{y y}^{(1,2, \ldots n)}=\left(\gamma_{1 y}^{2}+\gamma_{2 y}^{2}+\ldots \gamma_{n y}^{2}\right) S_{y y} .
$$

Thus, the multiple coherence is simply obtained by adding virtual coherences between the output and each virtual source component:

$$
\Gamma_{y}^{2}=\sum_{v=1}^{n} \gamma_{v y}^{2}
$$

\subsection{The Thresholded Multiple Coherence}

The classical multiple coherence, expressed by Eqs. (23) or (18), does not include the hypothesis test described on section 2.2. However, the thresholding step can easily be adapted to the MISO case considering Eq. (23). The multiple coherence being equal to a sum of virtual coherences, then it is straightforward to consider a summation of virtual coherences conditioned to their significance:

$$
\Gamma_{y, \alpha}^{2}=\sum_{v=1}^{n} \mathbf{1}_{\left[\gamma_{v y}^{2}>T\left(\alpha, N_{a p}\right)\right]} \cdot \gamma_{v y}^{2}
$$

where $\gamma_{v y}^{2}$ is expressed in Eq. (20) and where $T\left(\alpha, N_{a p}\right)$ is given by Eq. (9). A thresholded MCOP (TMCOP) can also be formulated based on the TMC:

$$
S_{y y, \alpha}^{(1,2 \ldots n)}=\Gamma_{y, \alpha}^{2} S_{y y} .
$$

Finally, when analysing the TMC for several outputs noted $y_{i}$, it can be interesting to formulate a global TMC function defining the global contribution of the inputs to the outputs (MIMO case). The global TMC can be simply 
defined as the ratio between the average output TMCOP and the average output spectra:

$$
\Gamma_{\alpha}^{2}=\frac{\sum_{i} \Gamma_{y_{i}, \alpha}^{2} S_{y_{i} y_{i}}}{\sum_{i} S_{y_{i} y_{i}}}
$$

\section{$3 \quad$ Numerical experiment}

A numerical experiment is conducted in order to illustrate the capability of the proposed approach in various situations. The general framework of the experiment is drawn in Fig. 4. A set of $m$ incoherent sources is mixed through

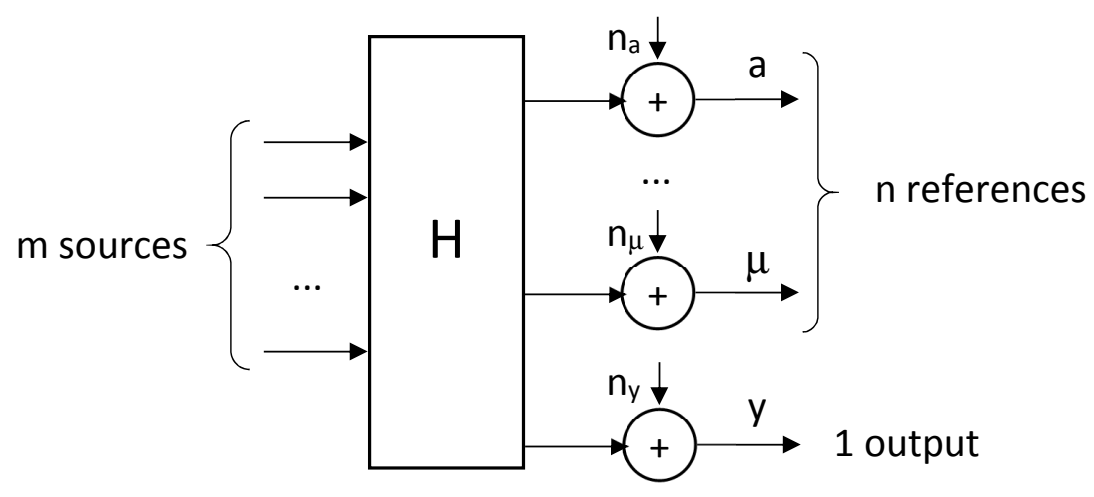

Fig. 4. General framework of the numerical experiment. $\mathrm{H}$ represents a $(n+1) \times m$ linear mixing matrix.

a linear mixing matrix $H$ so as to generate $n+1$ partially coherent signals. The $n$ first signals are considered as references, and the $n+1^{\text {th }}$ is the output. Incoherent noise is added to all generated signals, verifying given SNR values for references and for the output (noted $\mathrm{SNR}_{\text {ref }}$ and $\mathrm{SNR}_{\text {out }}$, respectively). The aim of the simulation is to estimate the contribution of the sources to the output signal, using the TMC. The estimated output SNR is directly linked to the multiple coherence value:

$$
\mathrm{SNR}_{\text {out }}=\frac{\Gamma_{y, \alpha}^{2}}{1-\Gamma_{y, \alpha}^{2}} .
$$

Simulations are implemented for $m=2$ (2 incoherent sources), $N_{0}=100$, a number of references equal to 1, 5 and 25 , and a reference $\mathrm{SNR}\left(\mathrm{SNR}_{r e f}\right)$ equal to $-10,0$ and $10 \mathrm{~dB}$. The multiple coherence is calculated including the hypothesis test proposed in this work for three values of $\alpha=100,5,1 \%$, 
$\alpha=100 \%$ being equivalent to the classical multiple coherence (threshold $T$ equal to 0 ). The number of incoherent sources (arbitrarily set to 2 for these
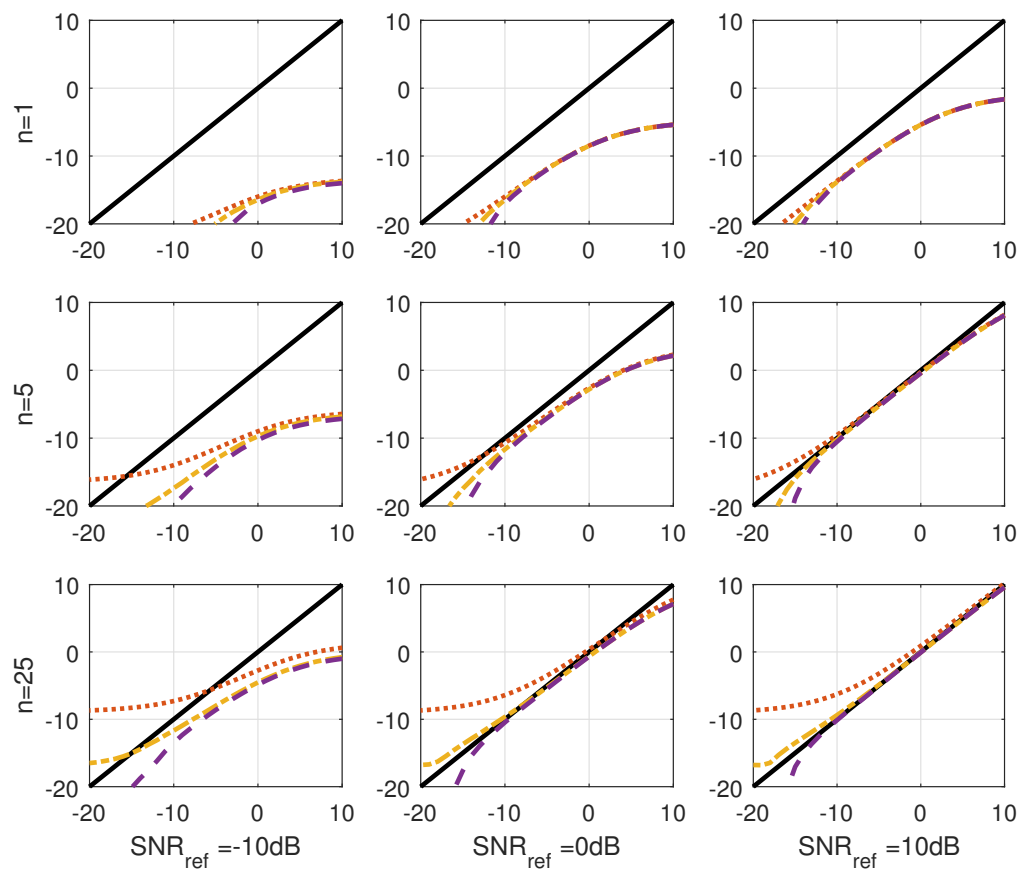

Fig. 5. Simulation results: $\mathrm{SNR}_{\text {out }}$ (the estimated one) as a function of $\mathrm{SNR}_{\text {out }}$ (the real one). Top to bottom : number of references equal to 1, 5, 25. Left to right : reference SNR set to $-10,0,10 \mathrm{~dB}$. Solid black : real $\mathrm{SNR}_{\text {out }}$. Color : SNR $\mathrm{R}_{\text {out }}$ with $\alpha=100 \%$ (no hypothesis test, dotted red) , $5 \%$ (dash-dotted yellow), $1 \%$ (dashed purple).

simulations) could have been another parameter of the study. However, the fact that the number of references should increase when the number of uncorrelated sources increases seems straightforward.

Results are given in Fig. 5, showing the estimated output SNR ( $\left.\mathrm{SNR}_{\text {out }}\right)$ obtained through Eq. (27) as a function of a real output SNR ( $\left.\mathrm{SNR}_{\text {out }}\right)$ varying between -20 and $10 \mathrm{~dB}$. When the estimated SNR is below (above) the $\mathrm{SNR}_{\text {out }}=\mathrm{SNR}_{\text {out }}$ line (solid black), it means that the SNR is under (resp. over) estimated.

Some general trends can be drawn from Fig. 5:

- when the number of references is too small, the output SNR is underestimated,

- the number of required references to get a correct estimate increases when the SNR of references decreases,

- when the reference SNR is poor and for positive output SNR values, the output SNR is systematically underestimated whatever the values of $\alpha$ and $n$, 
- for $\alpha=100 \%$, the estimated output SNR cannot go below a certain threshold increasing with the number of references (about $-15 \mathrm{~dB}$ for $n=5,-8$ $\mathrm{dB}$ for $n=25$ ), this effect is due to the statistical behaviour of the multiple coherence, discussed in $[21\rfloor$.

Also, it can be seen that when the number of references is high ( $n=25$ case), the output SNR becomes overestimated when the real output SNR is below $0 \mathrm{~dB}$. The interest of the thresholding approach proposed in this work is illustrated by curves obtained for $\alpha=5 \%, 1 \%$ when the number of references increases : it strongly attenuates the systematic overestimation observed with the classical approach. Even with a reference SNR at $0 \mathrm{~dB}$, the output SNR is correctly estimated up to $-15 \mathrm{~dB}$. With a very poor reference SNR $(-10 \mathrm{~dB}$ case), the output SNR remains slightly underestimated even with $n=25$. In such an extreme situation, the number of references could be increased, as well as the total record length, in order to improve the results.

\section{Experimental Validation in laboratory conditions}

A validation of the TMC-based source separation is proposed in this section, in the frame of a laboratory experiment. The experimental setup has been implemented in the frame of the ADAPT EU project (Cleansky 2 joint undertaking). The aim of the experiment was to compare different approaches for the separation of acoustic and aerodynamic contributions in wall pressure measurements in a wind tunnel (see [23] for more details). One of the measurement devices used to measure the wall-pressure consisted in a thin PCB (Printed Circuit Board) equipped with 76 MEMS microphones mounted on its back side, communicating with the front side through submillimeter aperture pinholes. The front face, free of any electronic component, is exposed to a turbulent boundary layer (TBL) generated by a flow $(20 \mathrm{~m} / \mathrm{s})$ established in a wind tunnel $(25 \mathrm{~cm} \times 50 \mathrm{~cm}$ section). Three loudspeakers are mounted in the roof of the tunnel, fed by 2 uncorrelated signals, and are generating an acoustic field in the tunnel. A sketch of the setup is given in Fig. 6. The array microphones are thus exposed by two types of pressure fluctuation, the aerodynamic part caused by the TBL and the acoustic part resulting from loudspeaker contributions. Note that considering that the flow velocity is relatively low (Mach number 0.06), the convection effect on the acoustic part of the signals is neglected. The acoustic part is thus considered as almost the same for measurement configurations with or without flow.

Four reference sensors are placed on the back side of the plate, so as to be not contaminated by the TBL developed on the front side. Two of them are microphones, placed in a small baffled volume with foam, and the two others are accelerometers directly pasted on the back side of the PCB. These references are expected to be free of the flow noise, while being still exposed 


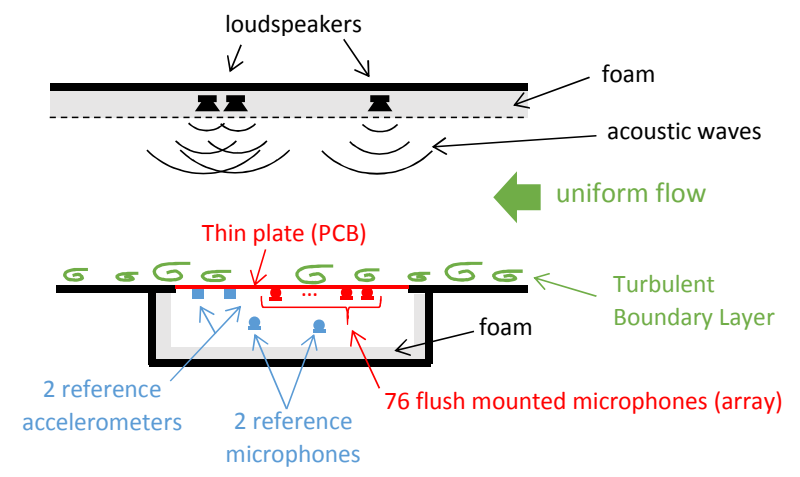

Fig. 6. General sketch of the wind tunnel experiment.

to the acoustic field crossing the plate because of its acoustic transparency. These assumptions can be verified by checking the power spectral densities of the different signals in configurations with or without flow (acoustic sources switched on in both cases). These spectra are shown in Fig. 7, averaged over the microphone array (left), over reference microphones (center) and over accelerometers (right).
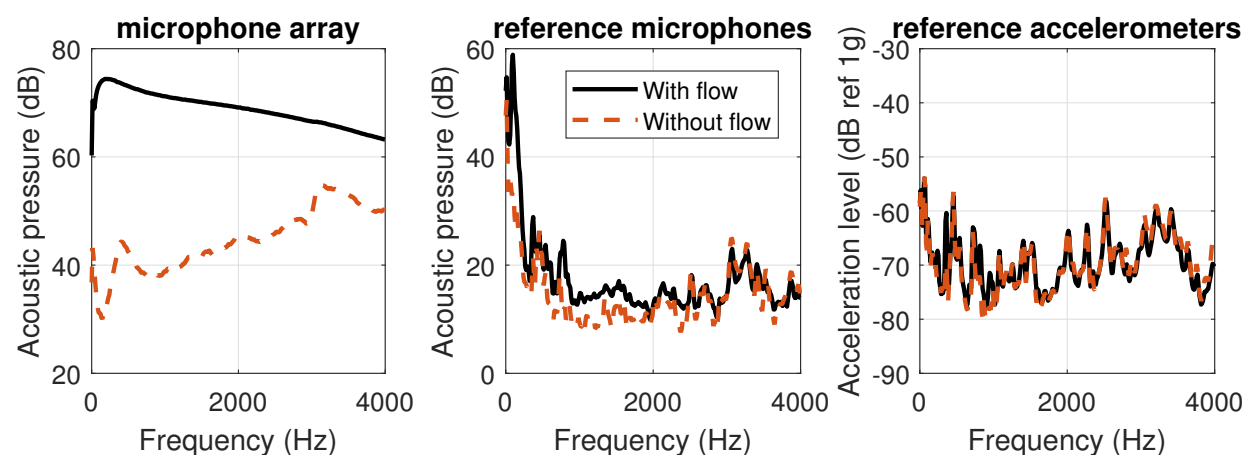

Fig. 7. Spectra obtained with (solid black) and without (dashed red) flow, acoustic sources switched on. Average spectra over the microphone array (left), reference microphones (center) and accelerometers (right).

It is clear that with the flow, the pressure measured by the microphone array essentially results from the aerodynamic pressure fluctuations generated by the TBL. The SNR (ratio between acoustic and aerodynamic pressure) falls between $-35 \mathrm{~dB}$ at low frequency and $-20 \mathrm{~dB}$ at high frequency. Conversely, the spectra of reference signals with and without flow are similar, which means that the SNR is much better than for the array microphones, especially for accelerometers. The differences observed on reference microphones are quite limited, except in the very low frequency range (up to $15 \mathrm{~dB}$ ) and at mid frequencies ( 3 to $5 \mathrm{~dB}$ ). Considering that the vibration of the plate is similar with or without flow, the differences observed on reference microphones may be due to the noise generated by the wind tunnel coming from the outside of the array backside cabinet. 
The ability of the PCB to filter out the TBL contribution is related to its thin-plate behaviour. The plate is made of glass-reinforced epoxy (FR-4), and is about $1 \mathrm{~mm}$ thick. According to the thin plate theory, such structures act as low pass filters in the wavenumber domain: high wavenumber components standing above the natural wavenumber of the structure are not able to efficiently excite it $[24]$. Considering the present experimental case, the convective wavenumber (carrying most of the TBL power) stands well above the natural wavenumber of the plate for the frequency domain that is above $100 \mathrm{~Hz}$. Conversely, the acoustic part of the exciting pressure field falls below the acoustic wavenumber, that stands below the natural wavenumber of the plate for frequencies below $20 \mathrm{kHz}$. The acoustic excitation is thus expected to be much more efficiently transmitted to the plate than the TBL part, in a wide frequency range from $100 \mathrm{~Hz}$ to $20 \mathrm{kHz}$.

The four reference signals are utilized hereafter so as to denoise array microphones, which means removing the aerodynamic noise contribution so as to recover the acoustic contribution. Note that recordings have been realized over time durations of $30 \mathrm{~s}$, and cross spectral quantities are averaged using time segments of $1 / 12 \mathrm{~s}$, with an overlap of $66 \%$, Hanning window (apparent number of snapshots $N_{a p}=745$ ). The average COP (Coherent Output Power) with flow is given in Fig 8 (left) for different values of $\alpha(100 \%, 5 \%$, $1 \%$ ), together with the total output power with flow. The total output power without flow is also drawn (dashed black), and considered as representative of the acoustic contribution with flow. The global TMC (Thresholded Multiple Coherence) is also drawn for the same values of $\alpha$ in the same figure (right). The global TMC being an estimation of the SNR, it is directly compared to the ratio between output powers measured without and with flow. Note that the first value of $\alpha=100 \%$ corresponds to the estimation of the classical multiple coherence function.

It can be clearly seen in Fig. 8 (right) that the classical multiple coherence overestimates the actual SNR on a wide frequency range $(0.5$ to $3 \mathrm{kHz})$. The overestimation factor lies between a bit more than 10 at $500 \mathrm{~Hz}$ and decreases to 2 only at about $2500 \mathrm{~Hz}$. The TMC estimation significantly reduces this estimation bias, the TMC with $\alpha=5 \%$ estimating quite well the actual SNR between 1500 and $2500 \mathrm{~Hz}$. The TMC with $\alpha=1 \%$ generally underestimates the SNR for the present experimental case. At low frequency $(f<500 \mathrm{~Hz})$, it is seen that for any value of $\alpha$, the TMC is strongly overestimated. This can be explained by the fact that the wind tunnel itself generates an acoustic background noise at low frequency. This noise, of acoustic nature, is efficiently transmitted to the PCB and thus contributes to reference signals. The hypothesis of incoherence between the noise affecting references and outputs is thus not satisfied, the TMC logically fails to identify the actual contribution of the acoustic source. 

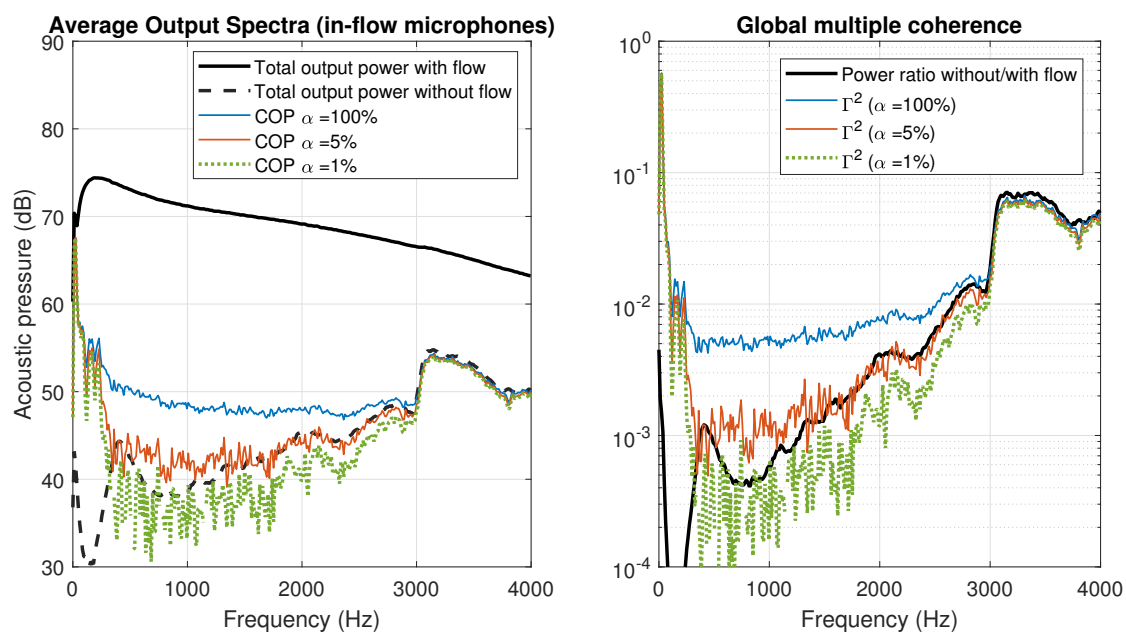

Fig. 8. Left: average spectra on the microphone array with (solid black) and without (dashed black) flow, acoustic sources switched on. In color : Global COP for different values of $\alpha$. Right : Output power ratio for array microphones without and with flow (solid black). In color : global TMC for different values of $\alpha$.

When looking at output spectra (same figure, left), the same observations can be made : the acoustic contribution is overestimated when using the classical multiple coherence, by up to $10 \mathrm{~dB}$. The use of the TMC strongly reduces this overestimation, except at low frequency as already discussed. Finally, it can be said that in this case the acoustic contribution is relatively well bounded by the COPs estimated with values of $\alpha$ equal to $5 \%$ (upper bound) and $1 \%$ (lower bound). However, this observation may not be interpreted as a general rule as it may depend on different parameters like the number of incoherent sources contributing to the signal, the number of references and the length of the recording (as it is discussed in section 3).

\section{Experimental application to aircraft flight tests}

Developments proposed in this work are finally applied to an industrial case, in order to analyse measurements recorded during aircraft flight tests. Considered signals are acquired from a set of 14 interior microphones (distributed in the passenger cabin) and a set of 35 external microphones (distributed along the fuselage, on one side of the plane, downstream to the wing). The sources of interest in this application consist in all noise sources that are located outside, mainly the engine (fan and jet) and the airframe, excluding the turbulent boundary layer (TBL) developed on the fuselage. External microphones are strongly disturbed by the latter, with a signal to noise ratio that can be extremely poor (negative values). Internal microphone records are composed of external source contributions transferred through the fuselage, and also of the contributions of several interior noise sources such as the ventilation 
system. A sketch of the experimental setup is drawn in Fig. 9.

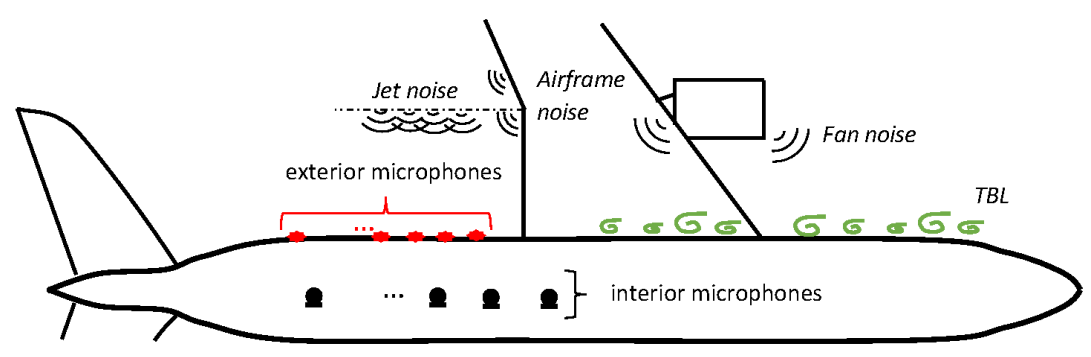

Fig. 9. Sketch of the flight test measurement setup.

Internal and external microphones are recorded simultaneously during stationary flight conditions. The engine is operating at a nominal condition, the Mach number is 0.85 . The total record length is $60 \mathrm{~s}$, the cross spectral matrix is calculated with a resolution of $4 \mathrm{~Hz}$ (Hanning window, overlap $67 \%$, $\left.N_{0}=240, N_{a p}=497\right)$.

\subsection{Contribution of external sources to interior noise}

A first TMC analysis can be implemented in order to determine the contribution of outside noise sources (excluding the TBL) to the interior noise. Note that the TBL is also a source strongly contributing to the interior noise, but it is distributed on the whole fuselage of the aircraft with very short correlation lengths, which makes it impossible to capture with only few reference sensors. Output signals are internal sensors, and references are external microphones distributed on the fuselage. The global TMC (Eq. (26)) is calculated for different values of $\alpha=100 \%, 5 \%$ and $1 \%(\alpha=100 \%$ corresponding to the classical approach without thresholding). Results are provided in Fig. 10, the global TMCOP on the left and the global TMC on the right. The global TMC can be interpreted here as the contribution of external sources (excluding TBL) located on the left side of the plane to interior noise, because external sensors that are used as references are distributed exclusively on the left side of the fuselage. This could explain why the global coherence never exceeds $50 \%$ (maximum global coherence at about 35 to $45 \%$ in the low frequency range). Assuming a symmetry between the contribution of external sources on both sides, this global coherence could be multiplied by 2 to account for the contribution of external sources on the right side. In mid and high frequency, the global multiple coherence decreases, and strong differences are observed for the different values of $\alpha$ used for the test. For $\alpha=100 \%$, the coherence never goes below about 7 or $8 \%$, illustrating the systematic 


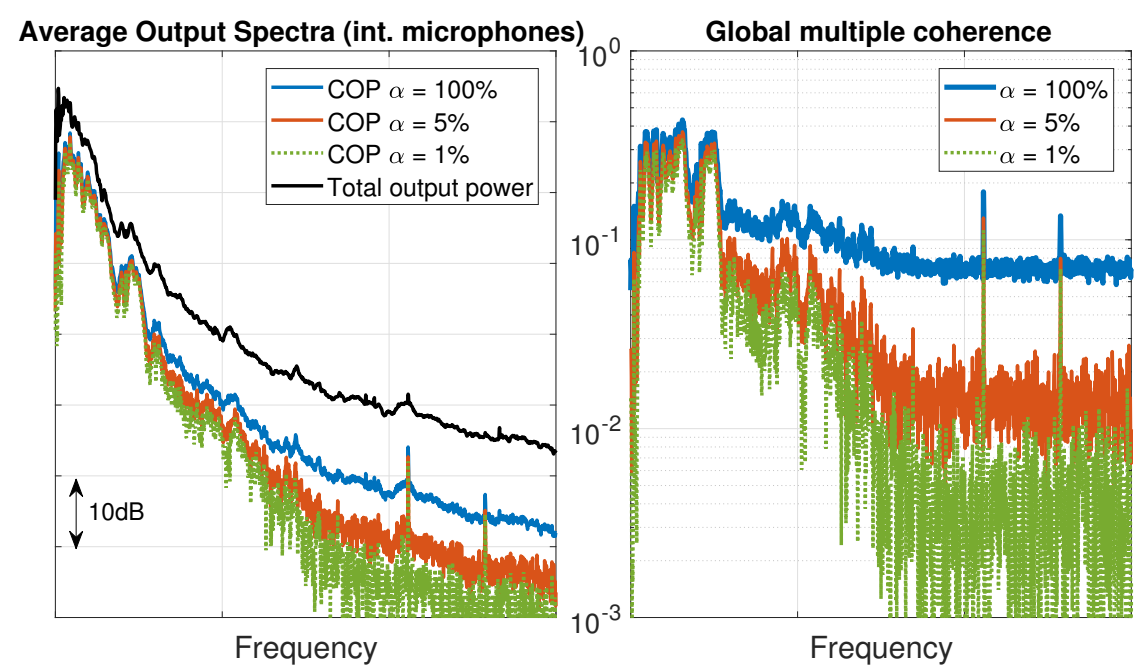

Fig. 10. Left : total (black) and Coherent (colours) output spectra with the classical approach $(\alpha=100 \%$, blue) and with $\alpha=5 \%-1 \%$ (red- green). Right: Global multiple coherence with the classical approach $(\alpha=100 \%$, blue) and with $\alpha=5 \%-1 \%$ (red - green).

overestimation effect of the classical multiple coherence already discussed in section 3 and in $[21]$. Using the proposed test for the thresholding of the virtual coherence, the multiple coherence drops to $2 \%(\alpha=5 \%)$ and even less than $1 \%(\alpha=1 \%)$. Some tonal components, that can be seen on either COPs or coherences, are emerging in the high frequency range. The emergence of these tonal components on microphones installed on the fuselage, as compared to the background noise of the COP, is strongly improved using the proposed approach : it is equal to 3 to $5 \mathrm{~dB}$ for $\alpha=100 \%$, and it increases to 8 to 10 $\mathrm{dB}$ and even to 12 to $15 \mathrm{~dB}$ for $\alpha=5 \%$ and $1 \%$, respectively.

\subsection{Denoising external microphones using internal references}

Another useful application of the TMC is proposed here, on the same dataset, but considering interior microphones as references, and exterior microphones as outputs. The aim of this operation is to try to remove as much as possible the TBL noise from fuselage microphones, so as to keep only the acoustical part of the measured pressure (note that the problem is very similar to the laboratory experiment reported in section 4 ). The acoustical part of the external pressure field is much more efficiently transmitted to the interior of the aircraft than the TBL noise. Indeed, the fuselage wall constitutes a low pass filter in the wavenumber domain, attenuating much more efficiently small wavelengths (TBL) than large wavelengths (acoustics). Moreover, the TBL noise inside the cabin results from sources distributed on the whole fuselage with short correlation lengths, so that the coherence between the TBL contribution to one 
external and one internal microphone is expected to be negligible. Internal microphones are thus used here as references to try to remove from external microphones the contribution of the TBL.

The global multiple coherence with or without the thresholding step are shown in Fig. 11 (right) together with the corresponding averaged COPs (left). A first observation is that the coherence values are much lower for this application than for the previous one (taking external microphones as references). This is logical, considering that the SNR of external microphones (strongly contaminated by the TBL) is expected to be quite low. The classical multiple coherence approach leads to a reduction of the broadband noise of about 15 $\mathrm{dB}$, and some tonal components, that are not seen on raw power spectra, are emerging by a few dBs. Considering results with the thresholding step, the high frequency broadband noise is much more reduced on COPs, by $20 \mathrm{~dB}$ $(\alpha=5 \%)$ and even $25 \mathrm{~dB}(\alpha=1 \%)$. The emergence of tonal components is also strongly increased, at least in the high frequency range, from 1 or $2 \mathrm{~dB}$ (classical approach) to 8 to $10 \mathrm{~dB}$ (with TMC).
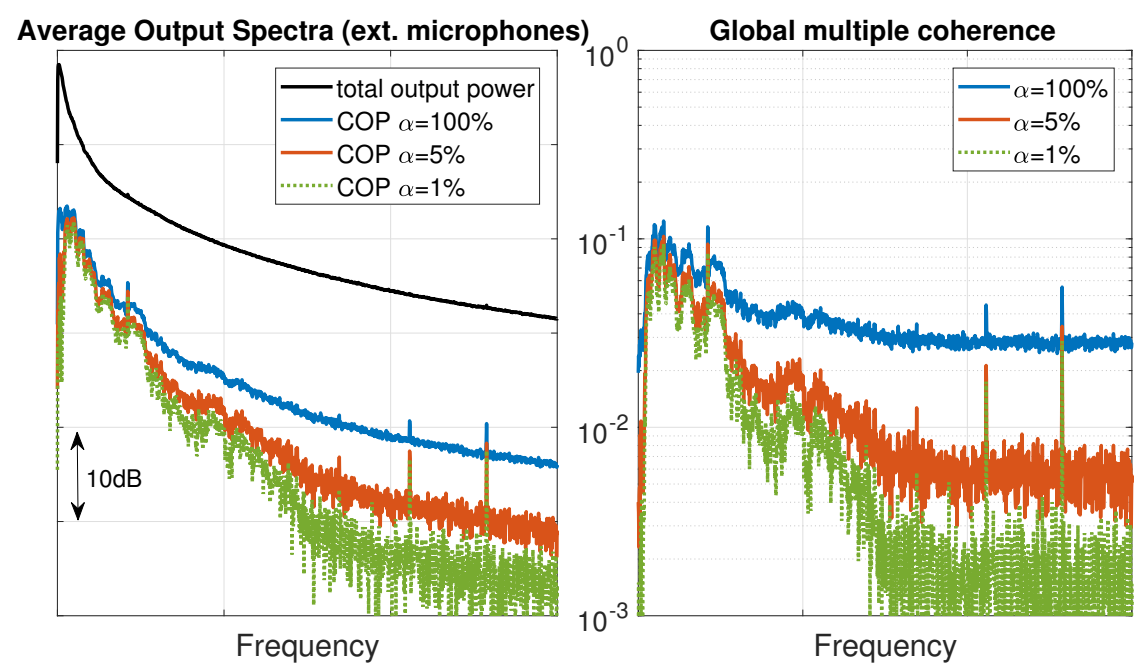

Fig. 11. Left : total (black) and Coherent (colours) output spectra with the classical approach $(\alpha=100 \%$, blue) and with $\alpha=5 \%-1 \%$ (red- green). Right : Global multiple coherence with the classical approach $(\alpha=100 \%$, blue) and with $\alpha=5 \%-1 \%$ (red - green).

\section{Conclusion}

This work proposes the implementation of a statistical test so as to put to zero an estimated coherence that can be considered as not significant. This thresholded coherence is used to estimate a corrected Coherent Output Power Spectrum, in order to get a better understanding of the significance of estimated source contributions in transfer path analysis approaches. This test 
has been extended to the multiple coherence (MISO / MIMO cases) through the use of the virtual source decomposition concept. Numerical simulations have shown that this test improves the estimation of source contributions especially if a large number of noisy references is used. An illustration in laboratory conditions has demonstrated the interest of the TMC (Thresholded Multiple Coherence) as compared to the classical multiple coherence, showing a significant improvement of the denoising possibilities. The interest of the Thresholded Multiple Coherence has finally been illustrated in the frame of an aircraft flight test experiment, either for the analysis of noise sources contributing to the cabin noise or for denoising fuselage microphones strongly disturbed by the turbulent boundary layer.

\section{Acknowledgements}

This work was performed within the framework of the Labex CeLyA of Université de Lyon, operated by the French National Research Agency (ANR-10LABX-0060/ANR-11-IDEX-0007), and of the Clean Sky 2 Joint Undertaking, European Union (EU), Horizon 2020, CS2-RIA, ADAPT project, Grant agreement no 754881.

\section{References}

[1] J.S. Bendat. Statistical errors in measurement of coherence functions and input/output quantities. Journal of Sound and Vibration, 59(3):405 - 421, 1978.

[2] P. Welch. The use of fast fourier transform for the estimation of power spectra: A method based on time averaging over short, modified periodograms. IEEE Transactions on Audio and Electroacoustics, 15(2):70-73, 1967.

[3] G. Carter. Receiver operating characteristics for a linearly thresholded coherence estimation detector. IEEE Transactions on Acoustics, Speech, and Signal Processing, 25(1):90-92, Feb 1977.

[4] Jeffrey Hilton Miles. Estimation of signal coherence threshold and concealed spectral lines applied to detection of turbofan engine combustion noise. The Journal of the Acoustical Society of America, 129(5):3068-3081, 2011.

[5] Clément Gallet and Claude Julien. The significance threshold for coherence when using the welch's periodogram method: Effect of overlapping segments. Biomedical Signal Processing and Control, 6(4):405 - 409, 2011.

[6] W. G. Halvorsen and J. S. Bendat. Noise source identification using coherent output power spectra. Sound and Vibration, 9(8):15, 18-24, 1975. 
[7] C.J. Dodds and J.D. Robson. Partial coherence in multivariate random process. Journal of Sound and Vibration, 42(2):243-249, 1975.

[8] J.S. Bendat. Solutions for the multiple input/output problem. Journal of Sound and Vibration, 44(3):311-325, 1976.

[9] J.S. Bendat and A.G. Piersol. Engineering applications of correlation and spectral analysis. Wiley-Interscience, New York, 1980.

[10] S.M. Price and R.J. Bernhard. Virtual coherence : A digital signal processing technique for incoherent source identification. In Proceedings of IMAC 4, Schenectady, NY, USA, 1986.

[11] N. Okubo and K. Kumazawa. Application of principal spectrum analysis to identify the vibration sources. In Proceedings of ISMA 13, Leuven, Belgium, 1988.

[12] M.S. Kompella, P. Davies, Bernhard R.J., and D.A. Ufford. A technique to determine the number of incoherent sources contributing to the response of a system. Mechanical System and Signal Processing, 8(4):363-380, 1994.

[13] P.R. Wagstaff, B. Bouizem, X. Bohineust, and J.C. Henrio. Source identification using partial coherence virtual coherence and principal inputs. In Proceedings of Inter-Noise 88, Avignon, France, 1988.

[14] C. Giangreco, P.R. Wagstaff, J.C. Henrio, and E. Langlois. Use of conditioned spectra to differentiate between flow noise and machinery noise. In Proceedings of Inter-Noise 95, Newport Beach, CA, USA, July 1995.

[15] M.F. Albright. Conditioned source analysis, a technique for multiple input system identification with application to combustion energy separation in piston engines. S.A.E. Technical paper series, (951376), 1995.

[16] Q. Leclere, C. Pezerat, B. Laulagnet, and L. Polac. Application of multi-channel spectral analysis to identify the source of a noise amplitude modulation in a diesel engine operating at idle. Applied Acoustics, 66:779-798, 2005.

[17] J. Y. Chung. Rejection of flow noise using a coherence function method. The Journal of the Acoustical Society of America, 62(2):388-395, 1977.

[18] B. N. Shivashankara. High bypass ratio engine noise component separation by coherence technique. Journal of Aircraft, 20(1), 1983.

[19] J. Hsu and K. Ahuja. A coherence-based technique to separate ejector internal mixing noise from farfield measurements. In proceedings of the 4 th AIAA/CEAS Aeroacoustics Conference, Toulouse,France, 1998.

[20] Ian Davis and Gareth Bennett. Experimental investigations of coherence based noise source identification techniques for turbomachinery applications - classic and novel techniques. In 17th AIAA/CEAS Aeroacoustics Conference 2011 (32nd AIAA Aeroacoustics Conference), 062011. 
[21] Q. Leclere, J. Antoni, E. Julliard, and A Pintado-Peno. Consideration of estimation error in multiple coherence approaches: application to the inside/ outside coherence analysis of aircraft flight tests. In proceedings of ISMA 2018, Leuven, Belgium, 2018.

[22] G. C. Carter and A. H. Nuttall. Statistics of the estimate of coherence. Proceedings of the IEEE, 60(4):465-466, April 1972.

[23] Q. Leclere, A. Dinsenmeyer, E. Salze, and J. Antoni. A comparison between different wall pressure measurement devices for the separation and analysis of tbl and acoustic contributions. In Proceedings of Flinovia III, Lyon, France, 2019 .

[24] Nicolas Totaro, Charles Pezerat, Quentin Leclère, Damien Lecoq, and Fabien Chevillotte. Identification of boundary pressure field exciting a plate under turbulent flow. In Flinovia-Flow Induced Noise and VIbration Issues and Aspects, pages 187-208. Springer, 2015. 